\title{
Intracellular siRNA delivery with novel spermine based surfactants
}

\author{
XU RongZuo ${ }^{1}$, WANG XuLi ${ }^{1} \&$ LU Zheng-Rong ${ }^{2 *}$ \\ ${ }^{1}$ Departments of Pharmaceutics and Pharmaceutical Chemistry, University of Utah, Salt Lake City, UT 84108, USA; \\ ${ }^{2}$ Department of Biomedical Engineering, Case Western Reserve University, Cleveland, OH 44106, USA
}

Received January 15, 2012; accepted May 10, 2012; published online August 16, 2012

\begin{abstract}
The design and development of safe and effective multifunctional siRNA delivery systems are critical for clinical application of RNAi therapeutics. Here we evaluated eight new spermine-based surfactant multifunctional carriers for siRNA delivery. These carriers complexed with siRNA forming stable compact nanoparticles with sizes around $100 \mathrm{~nm}$. The multifunctional carriers mediated higher intracellular siRNA transfection than Lipofectamine-2000. The siRNA nanoparticles of the multifunctional carriers exhibited low cytotoxicity as shown by MTT assay. Three of the eight multifunctional carriers showed higher silencing efficiency than Lipofectamine-2000 in both U87-Luc cells and CHO-GFP cells. SKAHCO showed the highest siRNA delivery efficiency among the carriers. It resulted in $84.6 \pm 5.5 \%$ silencing of luciferase activity in U87-Luc cells, much higher than that (62.8 $3.4 \%$ ) from Lipofectamine-2000. In conclusion, the spermine based multifunctional carriers are promising for highly efficient intracellular siRNA delivery.
\end{abstract}

RNAi, siRNA delivery systems, surfactant, nanoparticles, multifunctional carriers

Citation: $\quad$ Xu R Z, Wang X L, Lu Z R. Intracellular siRNA delivery with novel spermine based surfactants. Chin Sci Bull, 2012, 57: 3979-3984, doi: $10.1007 / \mathrm{s} 11434-012-5381-\mathrm{y}$

RNA interference (RNAi) is an emerging and promising therapeutic modality [1,2]. RNAi involves the degradation of an mRNA complementary to a small interfering RNA (siRNA) by a RNA-induced silencing complex (RISC) in mammalian cells. Any gene related to a human disease can be down-regulated by RNAi [3]. Clinical application of RNAi requires effective in vivo introduction of siRNA into the cells of interest, which still remains as the main challenge. siRNA can be encoded into plasmid DNA to deliver it to target cells with a viral or nonviral vector, but it is not a desirable approach [4,5]. Direct delivery of siRNA into cells is advantageous over siRNA encoding viral or plasmid DNA nonviral nanoparticles. siRNA can readily be synthesized by chemical methods in a large scale and high purity. It can also be chemically modified to improve its stability and efficacy [6-8]. Direct siRNA delivery avoids the complicated transcription procedure of a plasmid DNA and can induce RNAi activity in an efficient and controllable

*Corresponding author (email: zx1125@ case.edu) manner [3].

We have recently developed $\mathrm{pH}$-sensitive polymerizable surfactants as multifunctional carriers for nucleic acid delivery [9-11]. These carriers can form stable complexes with plasmid DNA or siRNA with sizes in the range of 100-200 nm. The carriers are composed of a $\mathrm{pH}$-sensitive amino head group, two distant lipid tails and two cysteinyl residues. With a packing parameter larger than 1, these carriers could transform into fusogenic inverse hexagonal conformation at acidic $\mathrm{pH}$ [12-14]. The protonation of the amino head group at $\mathrm{pH}$ lower than 6 could enhance the carriers' positive charge densities and their membrane disruption abilities due to $\mathrm{pH}$-sensitive amphiphilicity $[15,16]$. The thiol groups of the cysteinyl residues can be polymerized through autooxidation to form disulfide bonds to further stabilize the nanoparticles. These functions enable the carriers to facilitate endosomal escape of the nanoparticles and to release nucleic acids into cytosol via reduction of the disulfide bonds in the nanoparticles by cytosolic glutathione. The multifunctional carriers have demonstrated high intra- 
cellular and in vivo siRNA delivery efficiency, resulting in high intracellular gene silencing efficiency and therapeutic efficacy in a mouse tumor model $[10,11,17,18]$.

In a previous study, we reported a new series of multifunctional carriers based on spermine with modified structures and simplified synthetic procedure for the delivery of nucleic acids [19]. Spermine is a natural oligoamine that can condense nucleic acids via charge complexation [20,21]. The new multifunctional carriers demonstrated the ability of forming stable nanoparticles with plasmid DNA at low concentrations, the capability of efficient intracellular transfection of plasmid DNA and low cytotoxicity. In this study, we further investigated the effectiveness of these carriers for intracellular siRNA delivery. The nanoparticle formation between siRNA and the carriers was evaluated by dynamic light scattering. Intracellular siRNA delivery efficiency of these carriers was determined in CHO-GFP and U87-Luc cells with Lipofectamine-2000 as a control. The structure-activity relationship of these carriers for siRNA delivery was also evaluated.

\section{Materials and methods}

\subsection{Materials}

$\mathrm{N}$-(2,3-dioleoyloxy-1-propyl)trimethylammonium methyl sulfate (DOTAP), bovine serum albumin (BSA), and 2,5-diphenyl-3-(4,5-dimethyl-2-thiazolyl)tetrazolium bromide (MTT) were purchased from Sigma-Aldrich (St. Louis, MO, USA). The preparation of spermine based multifunctional carriers, SKCO, SKHCO, SHKCO, SHHKCO, SKACO, SKAHCO, SHKACO and SHHKACO, was reported in a previous literature [19]. The anti-Luc siRNA (sense sequence: 5'-CUUACGCUGAGUACUUCGAdTdT-3'; antisense sequence: 5'UCGAAGUACUCAGCGUAAdTdT-3') and anti-GFP siRNA (sense sequence: 5'-GCAAGCUGACCCUGAAGUUCAU-3'; antisense: 5'-GAACUUCAGGGUCAGCUUGCCG-3') were purchased from Qiagen (Valencia, CA, USA). The human glioma U87 MG cell line was purchased from American Type Culture Collection (ATCC, Manassas, VA, USA). The U87 cells constantly expressing luciferase were a gift from Dr. Randy Jansen of the University of Utah. CHO-EGFP cells expressing a green fluorescent protein were kindly provided by Dr. Charles Roth of Rugters University.

\subsection{Particle size measurement}

The siRNA nanoparticles were prepared by mixing siRNA solution with spermine based surfactants at predetermined $N / P$ ratios and incubated for $30 \mathrm{~min}$. The size of the nanoparticles was analyzed using a Brookhaven Instruments BI-200SM equipped with a $5 \mathrm{~mW}$ helium-neon laser with output at $633 \mathrm{~nm}$. Measurements were performed at room temperature in triplicates. The effective diameters of the nanoparticles were computed using the software provided by the manufacturer of the instrument.

\subsection{Surfactant mediated intracellular siRNA uptake}

Approximately $3 \times 10^{5}$ U87 cells per well were plated in 12 -well plates. After $24 \mathrm{~h}$, the nanoparticles, which were prepared by mixing $1 \mu \mathrm{g}$ AlexaFluor 488 labeled all negative siRNA (Qiagen) with each of multifunctional carriers at an $N / P$ ratio of 12 or Lipofectamine-2000 for $30 \mathrm{~min}$, were added into each well and incubated for $4 \mathrm{~h}$ at $37^{\circ} \mathrm{C}$ in $1 \mathrm{~mL}$ serum free medium. The medium was removed and the cells were washed twice with PBS and then trypsinized. The cells were collected and fixed by $4 \%$ paraformaldehyde in PBS for $20 \mathrm{~min}$. The samples were analyzed using a FACSCalibur flow cytometer (BD Bioscience) with FL-1 channel focused on the green fluorescence of AlexaFluor 488. Results were analyzed using Cell-Quest (BD Bioscience) software.

\subsection{Surfactants mediated silencing of luciferase with siRNA}

U87-Luc cells were seeded in 96 well plates at a density of $5 \times 10^{3}$ cells/well in DMEM medium containing $10 \%$ FBS, G418 $(300 \mu \mathrm{g} / \mathrm{mL})$, streptomycin $(100 \mu \mathrm{g} / \mathrm{mL})$ and penicillin (100 units/mL) $24 \mathrm{~h}$ prior to transfection. Anti-Luc siRNA was complexed with multifunctional carriers at an N/P ratio of 12 or Lipofectamine-2000 for $30 \mathrm{~min}$ and then incubated with cells at a siRNA concentration of $50 \mathrm{nmol} / \mathrm{L}$ for $4 \mathrm{~h}$ at $37^{\circ} \mathrm{C}$ in serum free DMEM medium. The medium was then replaced with complete DMEM medium and incubated for an additional $44 \mathrm{~h}$. Cells were washed with pre-warmed PBS, and treated with $200 \mu \mathrm{L} /$ well lysis buffer followed by a freeze-thaw cycle. Luciferase activity in cell lysates was then measured by a luciferase assay kit (Promega, Madison, WI, USA) on a luminometer (Dynex Tech., Chantilly, VA, USA). The gene silencing efficiency was normalized against the luciferase activity of untreated cells.

\subsection{Surfactants mediated GFP silencing with siRNA}

CHO-GFP cells were cultured in F12-K medium. Cells were seeded into 12 -well plates at the density of $2 \times 10^{5}$ cells per well and further cultured overnight. Nanoparticles formed by mixing $1.0 \mu \mathrm{g}$ anti-GFP siRNA with multifunctional carriers at an N/P ratio of 12 or Lipofectamine-2000 for $30 \mathrm{~min}$ at room temperature. The formed nanoparticles were then added to CHO-GFP cells in serum free F-12K medium at a siRNA concentration of $34 \mathrm{nmol} / \mathrm{L}$. After $4 \mathrm{~h}$ incubation at $37^{\circ} \mathrm{C}$, the medium was replaced with fresh complete F12-K medium and cultured for an additional $20 \mathrm{~h}$. Cells were then washed with pre-warmed PBS, harvested through trypsinization, and fixed in cold PBS containing 4\% paraformaldehyde for $20 \mathrm{~min}$. Samples were analyzed using a FACSCalibur flow cytometer with FL-1 channel focused on green fluorescence. Results were analyzed using Cell-Quest software. 


\subsection{Cytotoxicity study of siRNA/multifunctional carri- er nanoparticles}

The cytotoxicity of siRNA/multifunctional carrier nanoparticles was evaluated by an MTT assay in U87 cells. Briefly, U87 cells were plated in 96 -well plates with $1 \times 10^{4}$ cells per well and cultured overnight. Anti-Luc siRNA (10 pmol) was mixed with multifunctional carriers at an $N / P$ ratio of 12 or Lipofectamine-2000 at a predetermined dose and incubated at room temperature for $30 \mathrm{~min}$ to form nanoparticles. The formed nanoparticles were then added into serumfree MEM medium to reach a final concentration of $50 \mathrm{nmol} / \mathrm{L}$ siRNA and incubated with U87 cells for $4 \mathrm{~h}$. The medium was then replaced with complete MEM medium and continued culturing for an additional $20 \mathrm{~h}$. The medium was removed and cells were digested by $200 \mu \mathrm{L}$ DMSO. The absorption was read at $570 \mathrm{~nm}$ using a plate reader (Model 680, Bio-Rad, Hercules, CA, USA). The relative cell viability was calculated by the equation: ([Abs]sample-[Abs]blank)/ ([Abs]control-[Abs]blank) $\times 100 \%$.

\section{Results and discussion}

The chemical structures of the spermine-based surfactants,
SKCO, SKHCO, SHKCO, SHHKCO, SKACO, SKAHCO, SHKACO and SHHKACO, are shown in Figure 1. All of spermine based multifunctional carriers formed stable nanoparticles with siRNA at low concentrations. The size of nanoparticles at $N / P$ ratio 12 was in the range of $85.5 \mathrm{~nm}$ (SKHCO) and $120.6 \mathrm{~nm}$ (SKAHCO) as determined by dynamic light scattering (Figure 2). The combination of charge complexation of siRNA and the spermine head group, hydrophobic condensation of oleoyl lipid tails and oxidation of the dithiols resulted in the formation of compact siRNA nanoparticles.

The spermine based multifunctional carriers mediated high intracellular delivery efficiency of AlexaFluor 488 labeled siRNA. Figure 3 shows flow cytometric diagrams of the uptake of the labeled siRNA in U87 cells mediated by various carriers at an N/P ratio of 12 . The multifunctional carriers resulted in approximately $91 \%$ to $96 \%$ siRNA transfection in U87 cells, while Lipofectamine-2000 only transfected $73 \%$ of cells under the same condition. It appears that the carriers SKHCO, SKCO and SHHKCO with a lysine "Y" joint were lightly more effective intracellular siRNA delivery than the corresponding SKAKCO, SKACO and SHHKACO with a lysyl- $\beta$-alanine joint.

Figure 4 shows the efficiency of silencing luciferase expression in U87-Luc cells by an anti-Luc siRNA mediated<smiles>[R]C(=O)NC(CS)C(=O)NC(CS)C(=O)NC(CS)C(=O)NC(CS)C([R])=O</smiles><smiles>[R]C(=O)N[C@@H](CS)C(=O)NCCCCC(NC(=O)[C@H](CS)NC([R2])=O)C(=O)N[C@@H](Cc1cnc[nH]1)C([R])=O</smiles><smiles>[R]C(=O)NC(CS)C(=O)NCCCCC(NC(=O)CCNC(=O)C(CS)NC(Cc1cnc[nH]1)C([R])=O)C(=O)NC([R])CS</smiles>

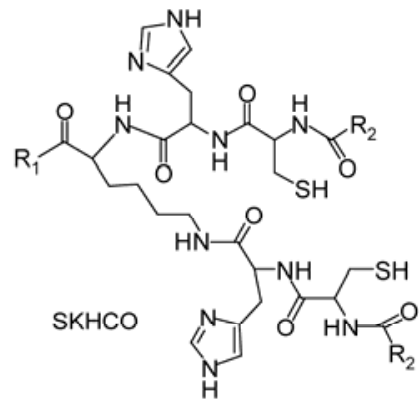<smiles>[R2]C(=O)NC(CCCCNC(=O)[C@H](CS)NC([R])=O)C(=O)NCCC(=O)N[C@@H](CS)C([R])=O</smiles>
SKACO<smiles>[R]C(=O)NC(CS)C(=O)NCCCCC(NC(=O)CCNC(=O)C(CS)NC([R])=O)C(=O)NC(=O)C(Cc1cnc[nH]1)NC(=O)C(Cc1c[nH]cn1)NC([R])=O</smiles><smiles>[R]C(=O)NC(CS)C(=O)NC(CS)C(=O)NC(CCCCNC(=O)[C@H](Cc1cnc[nH]1)NC([R2])C([R])=O)C(=O)N[C@@H](Cc1c[nH]cn1)C([R])=O</smiles><smiles>[R]C(=O)NC(CS)C(=O)NC(Cc1c[nH]cn1)C(=O)NCCC(=O)NC(CS)C(=O)NC(Cc1c[nH]cn1)C(=O)NCCCCNC(=O)C(CS)NC([R])C([R])=O</smiles><smiles></smiles><smiles>[R]=C=CCCCCCC#CCCCCCCCCC</smiles>

Figure 1 Chemical structures of the spermine based lipid surfactants. 


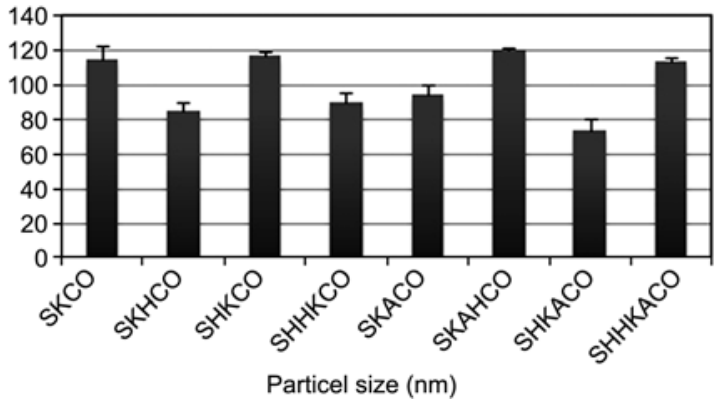

Figure 2 The size of the siRNA nanoparticles with the spermine based multifunctional carriers at an $N / P$ ratio of $12(n=3)$. by the carriers. SKHCO, SHHKCO, SKAHCO, SKACO and SHKACO resulted in higher luciferase silencing efficiency than Lipofectamine-2000. Among these carriers, SKAHCO showed the highest gene silencing efficiency $(84.6 \pm 5.5 \%)$ at $50 \mathrm{nmol} / \mathrm{L}$ siRNA and $N / P$ ratio of 12 . The gene silencing efficiency mediated by the carriers was further determined in CHO-GFP cells with an anti-GFP siRNA. Figure 5 shows flow cytometric diagrams of CHO-GFP cells after the treatment with the nanoparticles of an anti-GFP siRNA and various carriers. SKAHCO at $N / P$ ratio 12 again mediated the highest GFP silencing efficiency (61\%). SHKACO (49\%), SKHCO (49\%) and SKACO (51\%) also resulted in better
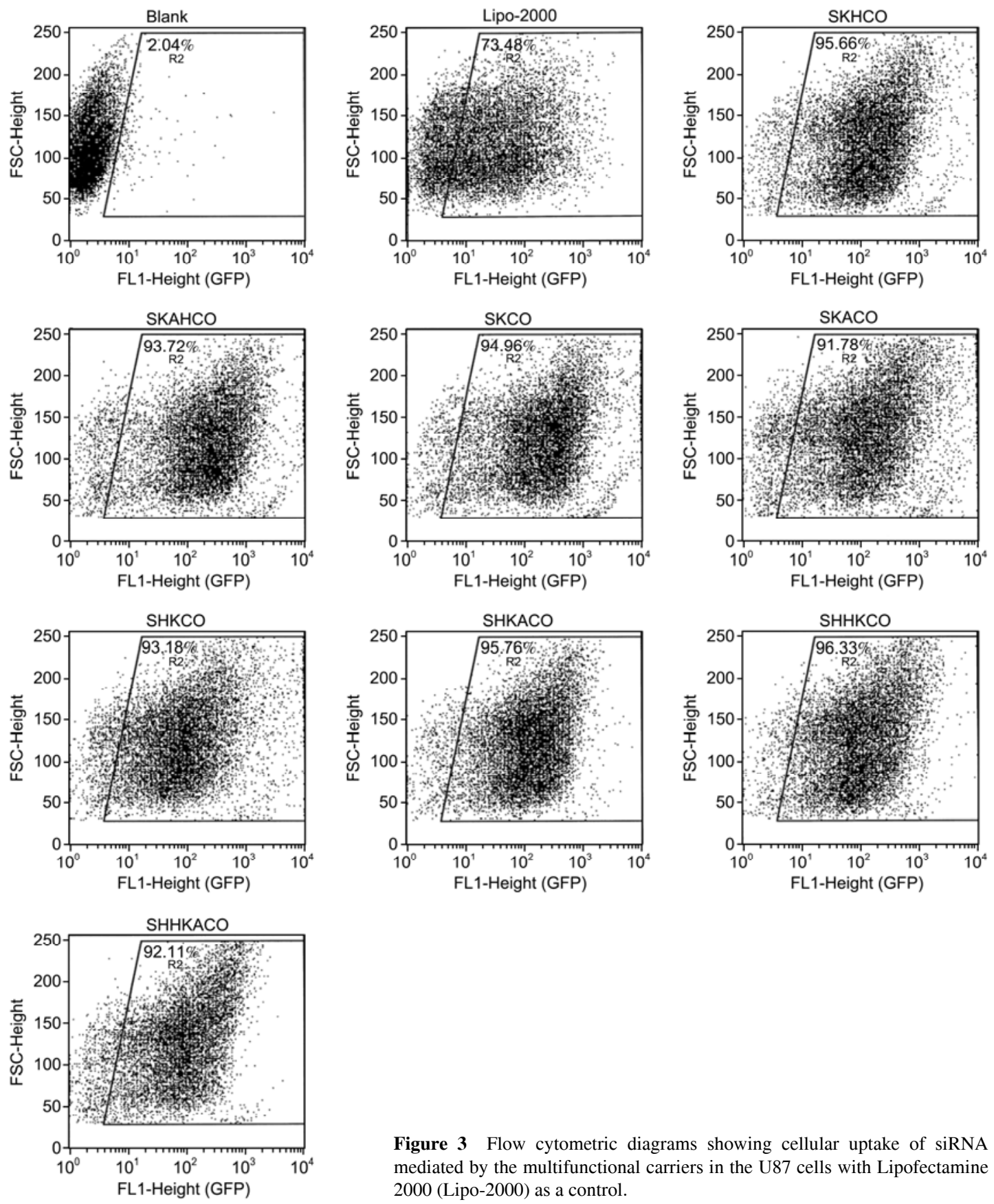

Figure 3 Flow cytometric diagrams showing cellular uptake of siRNA mediated by the multifunctional carriers in the U87 cells with Lipofectamine 2000 (Lipo-2000) as a control. 


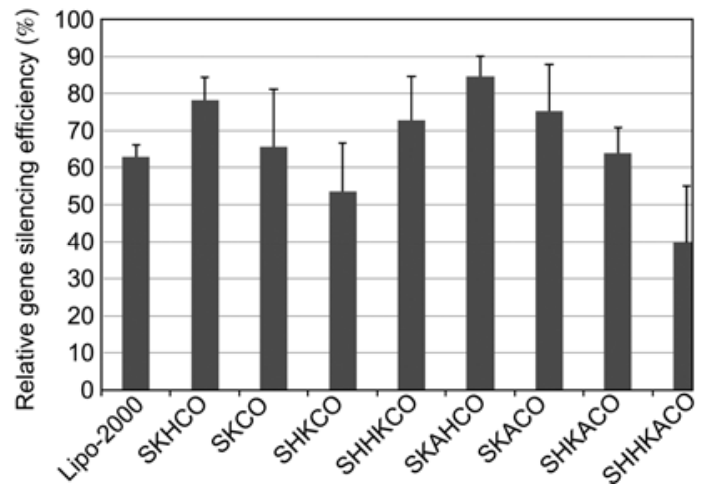

Figure 4 Silencing efficiency of luciferase expression in U87-Luc cells mediated by the nanoparticles of an anti-Luc siRNA $(50 \mathrm{nmol} / \mathrm{L})$ with the multifunctional carriers. The relative gene silencing efficiency was normalized to untreated cells.
GFP silencing than Lipofectamine-2000 (40\%).

Figure 6 shows the viability of U87 cells treated with the siRNA nanoparticles of the spermine based siRNA nanoparticles at N/P of 12. The MTT assay showed that the siRNA nanoparticles of the carriers resulted in low cytotoxicity at $50 \mathrm{nmol} / \mathrm{L}$ siRNA. The SKAHCO/siRNA nanoparticles with the highest gene silencing efficiency caused little cytotoxicity. The cytotoxicity of the siRNA nanoparticles of the multifunctional carriers was comparable with that of the siRNA complexes of Lipofectamine-2000.

The use of RNAi to treat human diseases requires the delivery of therapeutic siRNA into target cells. The design and development of safe and effective siRNA delivery system is a key step towards to clinical application of RNAi therapeutics. The delivery systems should be able to protect siRNA from
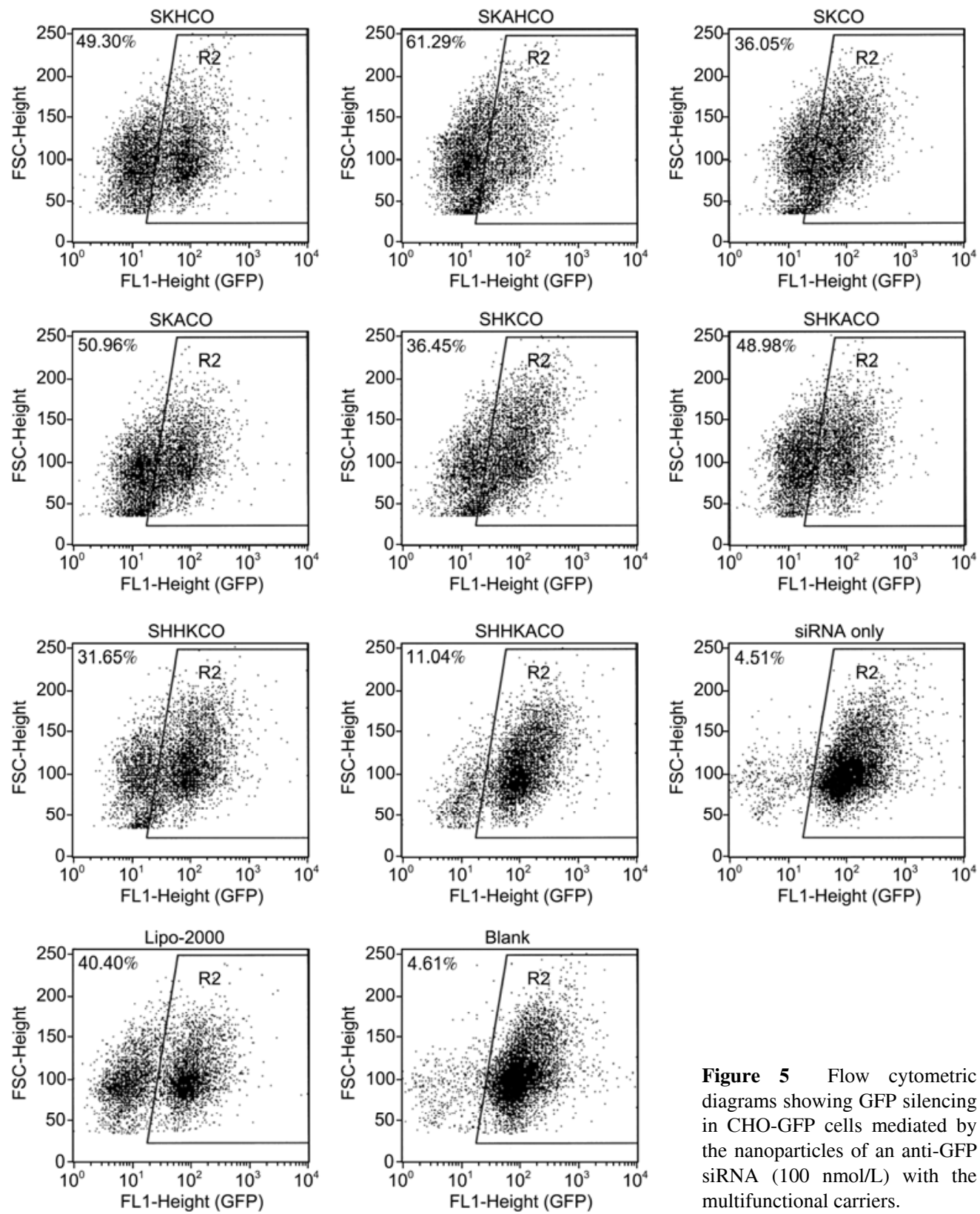

Figure 5 Flow cytometric diagrams showing GFP silencing in CHO-GFP cells mediated by the nanoparticles of an anti-GFP siRNA $(100 \mathrm{nmol} / \mathrm{L})$ with the multifunctional carriers. 


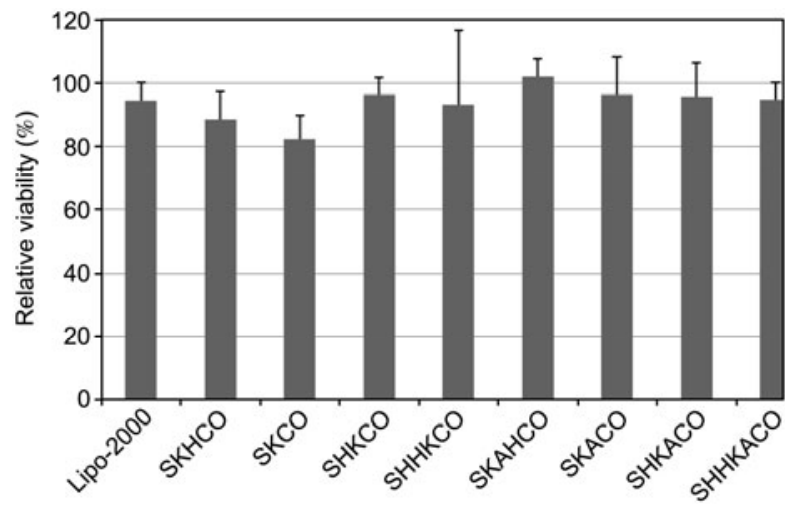

Figure 6 Cytotoxicity of the siRNA nanoparticles with the multifunctional carriers at an N/P ratio of 12 in U87 cells. siRNA concentration was $50 \mathrm{nmol} / \mathrm{L}$. Lipofectamine 2000 (Lipo-2000) was used as control.

enzymatic degradation during delivery process [22], to deliver siRNA into targets cells and to release it into cytosol. In this study, we evaluated the efficacy of eight new spermine based surfactants as the multifunctional carriers for siRNA delivery. These carriers were able to form stable and compact nanoparticles with siRNA and resulted in high intracellular siRNA uptake. Six carriers contained one or two histidine residuals. The imidazole group in histidine had a $\mathrm{p} K_{\mathrm{a}}$ around 6.5 and was expected to facilitate endosomal escape of the siRNA nanoparticles at endosomal $\mathrm{pH}$ for enhanced gene silencing. However, SHHKACO with two histidine residues mediated the lowest gene silencing efficiency in both U87-Luc and CHO-GFP cells among the carriers. The other histidine containing carriers did not have any advantage over the carriers without histidine, SKACO and SKCO. The carriers SKACO, SKAHCO, SHKACO and SHHKACO had a more symmetric location of lipid tails than SKCO, SKHCO, SHKCO and SHHKCO. It appears that the location of the lipid tails did not have a significant impact on intracellular siRNA uptake and gene silencing efficiency mediated by the surfactants. Nevertheless, three carriers, SKAHCO, SKHCO and SKACO, were more effective than Lipofectamine-2000 in silencing the expression of both luciferase and GFP. Overall, SKACO demonstrated the highest intracellular plasmid DNA delivery efficiency as shown in the previous study [19] and SKAHCO showed the highest siRNA delivery efficiency.

\section{Conclusion}

The spermine based surfactants formed stable nanoparticles with siRNA at an N/P ratio of 12 . The nanoparticles had relatively low cytotoxicity and resulted in high intracellular uptake of AlexaFluor488-labeled siRNA. Five of these carriers possessed a higher overall luciferase activity knockdown efficiency than Lipofectamine-200 when delivering
anti-Luc siRNA. Four of these multifunctional carriers showed a higher GFP silencing efficiency than Lipofectamine-2000. Among these tested carriers, SKAHCO demonstrated the highest intracellular siRNA delivery efficiency. It has a great potential for safe and effective siRNA delivery.

1 Aagaard L, Rossi J J. RNAi therapeutics: Principles, prospects and challenges. Adv Drug Deliv Rev, 2007, 59: 75-86

2 Tiemann K, Rossi J J. RNAi-based therapeutics-current status, challenges and prospects. EMBO Mol Med, 2009, 1: 142-151

3 Fire A, Xu S, Montgomery M K, et al. Potent and specific genetic interference by double-stranded RNA in Caenorhabditis elegans. Nature, 1998, 391: 806-811

4 Wilson J A, Richardson C D. Induction of RNA interference using short interfering RNA expression vectors in cell culture and animal systems. Curr Opin Mol Ther, 2003, 5: 389-396

5 Lai S R, Andrews L G, Tollefsbol T O. RNA interference using a plasmid construct expressing short-hairpin RNA. Methods Mol Biol, 2008, 405: 31-37

6 Bramsen J B, Kjems J. Chemical modification of small interfering RNA. Methods Mol Biol, 2011, 721: 77-103

7 Gaglione M, Messere A. Recent progress in chemically modified siRNAs. Mini Rev Med Chem, 2010, 10: 578-595

8 Chernolovskaya E L, Zenkova M A. Chemical modification of siRNA. Curr Opin Mol Ther, 2010, 12: 158-167

9 Xu R, Wang X L, Lu Z R. New amphiphilic carriers forming $\mathrm{pH}$-sensitive nanoparticles for nucleic acid delivery. Langmuir, 2010, 26: 13874-13882

10 Wang X L, Nguyen T, Gillespie D, et al. A multifunctional and reversibly polymerizable carrier for efficient siRNA delivery. Biomaterials, 2008, 29: 15-22

11 Wang X L, Ramusovic S, Nguyen T, et al. Novel polymerizable surfactants with $\mathrm{pH}$-sensitive amphiphilicity and cell membrane disruption for efficient siRNA delivery. Bioconjug Chem, 2007, 18: 2169-2177

12 Bell P C, Bergsma M, Dolbnya I P, et al. Transfection mediated by gemini surfactants: Engineered escape from the endosomal compartment. J Am Chem Soc, 2003, 125: 1551-1558

13 Koltover I, Salditt T, Radler J O, et al. An inverted hexagonal phase of cationic liposome-DNA complexes related to DNA release and delivery. Science, 1998, 281: 78-81

14 Semple S C, Akinc A, Chen J, et al. Rational design of cationic lipids for siRNA delivery. Nat Biotechnol, 2010, 28: 172-176

$15 \mathrm{Xu}$ Y, Szoka F C Jr. Mechanism of DNA release from cationic liposome/DNA complexes used in cell transfection. Biochemistry, 1996, 35: 5616-5623

16 Singh R S, Goncalves C, Sandrin P, et al. On the gene delivery efficacies of $\mathrm{pH}$-sensitive cationic lipids via endosomal protonation: A chemical biology investigation. Chem Biol, 2004, 11: 713-723

17 Wang X L, Xu R, Lu Z R. A peptide-targeted delivery system with $\mathrm{pH}$-sensitive amphiphilic cell membrane disruption for efficient receptor-mediated siRNA delivery. J Control Release, 2009, 134: 207-213

18 Wang X L, Xu R, Wu X, et al. Targeted systemic delivery of a therapeutic siRNA with a multifunctional carrier controls tumor proliferation in mice. Mol Pharm, 2009, 6: 738-746

$19 \mathrm{Xu} \mathrm{R}, \mathrm{Lu} \mathrm{Z}$ R. Design, synthesis and evaluation of spermine-based $\mathrm{pH}$-sensitive amphiphilic gene delivery systems: Multifunctional non- viral carriers. Sci China Chem, 2011, 54: 1-10

20 Behr J P, Demeneix B, Loeffler J P, et al. Efficient gene transfer into mammalian primary endocrine cells with lipopolyamine-coated DNA. Proc Natl Acad Sci USA, 1989, 86: 6982-6986

21 Remy J S, Sirlin C, Vierling P, et al. Gene transfer with a series of lipophilic DNA-binding molecules. Bioconjug Chem, 1994, 5: 647-654

22 Keller M. Lipidic carriers of RNA/DNA oligonucleotides and polynucleotides: What a difference a formulation makes! J Control Release, 2005, 103: 537-540

Open Access This article is distributed under the terms of the Creative Commons Attribution License which permits any use, distribution, and reproduction in any medium, provided the original author(s) and source are credited. 